\title{
Genome-Wide Transcriptional Response During the Development of Bleomycin-Induced Pulmonary Fibrosis in Sprague-Dawley Rats
}

\author{
Han-Jin Park', Mi-Jin Yang ${ }^{2}$, Jung-Hwa Oh', Young-Su Yang ${ }^{2}$, Myung-Sang Kwon', \\ Chang Woo Song ${ }^{2}$ and Seokjoo Yoon ${ }^{1}$ \\ ${ }^{1}$ Division of Research and Development, Korea Institute of Toxicology, Daejeon 305-343 \\ ${ }^{2}$ Division of Inhalation Toxicology, KIT Jeongeup Campus, Jeollabuk-do 580-185, Korea
}

(Received February 22, 2010; Revised April 2, 2010; Accepted April 6, 2010)

\begin{abstract}
Pulmonary fibrosis is a common consequence of many lung diseases and a leading cause of morbidity and mortality. The molecular mechanisms underlying the development of pulmonary fibrosis remain poorly understood. One model used successfully to study pulmonary fibrosis over the past few decades is the bleomycin-induced pulmonary fibrosis model. We aimed to identify the genes associated with fibrogenesis using an Affymetrix GeneChip system in a bleomycin-induced rat model for pulmonary fibrosis. To confirm fibrosis development, several analyses were performed, including cellular evaluations using bronchoalveolar lavage fluid, measurement of lactate dehydrogenase activity, and histopathological examinations. Common aspects of pulmonary fibrosis such as prolonged inflammation, immune cell infiltration, emergence of fibroblasts, and deposition of extracellular matrix and connective tissue elements were observed. Global gene expression analysis revealed significantly altered expression of genes $(\geq 1.5$-fold, $p<0.05$. $)$ in a time-dependent manner during the development of pulmonary fibrosis. Our results are consistent with previous results of well-documented gene expression. Interestingly, the expression of triggering receptor expressed on myeloid cells 2 (Trem2), secreted phosphoprotein 1 (Sppl), and several proteases such as Tpsab1, Mcpt1, and Cmal was considerably induced in the lung after bleomycin treatment, despite little evidence that they are involved in pulmonary fibrogenesis. These data will aid in our understanding of fibrogenic mechanisms and contribute to the identification of candidate biomarkers of fibrotic disease development.
\end{abstract}

Key words: Bleomycin, Gene expression, Lung fibrosis

\section{INTRODUCTION}

Idiopathic pulmonary fibrosis is a consequence of abnormal tissue repair, which is characterized by extensive inflammation in the interstitial and alveolar spaces, proliferation of fibroblasts, and progressive fibrosis leading to the destruction of lung function (Selman et al., 2004). Pulmonary fibrosis involves cytokine networks and cellular interactions among several cell types, which results in increased collagen gene expression and collagen deposition in the lungs (Piguet et al., 1990; Gharaee-Kermani et al., 2005). Despite many investigations on the pathogenesis of pulmonary fibrosis and its relationship to immune cells, extracellular matrix repair, cytokines, and chemokines, the molecular

Correspondence to: Seokjoo Yoon, Toxicogenomics Team, Korea Institute of Toxicology, 19 Shinsung-ro, Yuseong, Daejeon 305-343, Korea

E-mail: sjyoon@kitox.re.kr mechanisms underlying this disease remain unclear (Zhang et al., 1994, 1996; Kuwano et al., 2001; Gharaee-kermani et al., 2008).

Bleomycin is a useful chemotherapeutic antitumor drug, derived from Streptomyces verticillus; however, as a side effect, bleomycin treatment may lead to pulmonary fibrosis in humans and animals. Therefore, animal models of pulmonary fibrosis induced by the intratracheal instillation of bleomycin have been well established and widely used for studying the mechanisms underlying fibrosis and potential therapeutic agents (Jordana et al., 1988; Sakanashi et al., 1994; Gharaee-Kermani et al., 2005; Moeller et al., 2008). Several studies using microarrays have been performed to evaluate gene expression patterns in the lungs after bleomycin instillation and identify the major genes involved in the progression of pulmonary fibrosis (Kaminski et al., 2000; Zuo et al., 2002; Katsuma et al., 2001; Pottier et al., 2007). These studies have provided information about genes that play central roles in the progression of fibrosis over time in 
a mouse model. Although genomic approaches using bleomycin-induced pulmonary fibrosis animal models have been useful for understanding the molecular mechanisms underlying fibrosis and for identifying fibrogenic markers, these approaches have limitations related to the vast data sets generated and the difficulty of replicating progressive fibrosis in animal models. Furthermore, recent studies aimed at elucidating fibrogenesis mechanisms and developing therapeutic agents for the treatment of fibrosis have focused on mouse models because of the convenience of using genetically modified animals.

Here, we performed a microarray-based gene expression analysis to reveal distinct gene expression patterns and to identify genes associated with the progression of pulmonary fibrosis in the lungs of Sprague-Dawley (SD) rats at 7, 14 , and 28 days after treatment with bleomycin by intratracheal instillation (ITI). Lung damage and pulmonary fibrogenesis were assessed by cellular evaluation of bronchoalveolar lavage fluid (BALF), measurement of lactate dehydrogenase (LDH) activity, and histopathological examination of lung tissue.

\section{MATERIALS AND METHODS}

Animals and experimental design. Pathogen-free, 7week-old, male Sprague-Dawley (SD) rats (Orient Bio Inc., Korea), weighing 210 to 240 g, were administered bleomycin hydrochloride (Nippon Kayaku, Tokyo, Japan) as a single dose of $2.5 \mathrm{mg} / \mathrm{kg}$ body weight, dissolved in $0.1 \mathrm{~m} l$ of saline $(0.9 \% \mathrm{NaCl})$, via intratracheal instillation (ITI) under isoflurane anesthesia. Control rats received an equal volume of saline. The dose of bleomycin was selected based on previous experiments in our laboratory and is known to cause consistent biochemical and histological damage, but not mortality (Cortijo et al., 2001).

The treated and control rats were randomly assigned to six weight-matched groups $(n=5$ for each): three treated groups (7, 14, and 28 days), and three corresponding control groups $(7,14$, and 28 days). On days 7, 14, and 28 following ITI bleomycin or saline, the rats were sacrificed by exsanguination via the abdominal aorta, under an overdose of isoflurane anesthesia. Lung injury was evaluated by analysis of bronchoalveolar lavage fluid (BALF) and light microscopic examination (Olympus, Japan).

All experiments were approved by the Institutional Animal Care and Use Committee and conducted in accordance with Association for Assessment and Accreditation of Laboratory Animal Care international guidelines.

Analysis of bronchoalveolar lavage fluid (BALF). After exsanguination, the left lung of each rat was clamped off, the right lung was washed twice with $0.9 \%$ saline, and the BALF was collected. The cell-free supernatant obtained by centrifugation $\left(500 \mathrm{~g}, 10 \mathrm{~min}, 4^{\circ} \mathrm{C}\right)$ of the BALF sample was analyzed for LDH activity, as described below. The right lung was lavaged twice more with $3 \mathrm{~m} l$ of saline, and the cell pellet was recovered after centrifugation $(500 \mathrm{~g}, 10 \mathrm{~min}$, $4^{\circ} \mathrm{C}$ ) of this sample. The cell pellets from the rats were combined for each group, resuspended in $1 \mathrm{~m} l$ of saline, and used to evaluate cellular parameters.

The cells recovered in the BALF pellet were identified and counted. Total cell number was determined using a cell viability analyzer (Beckman Coulter, Fullerton, CA). To collect the BAL cells on a slide, a sample was spun at $800 \mathrm{rpm}$ for $5 \mathrm{~min}$ in a Cytospin 4 centrifuge (Thermo-Shandon, Pittsburgh, PA). The cells were stained with Wright-Giemsa Sure stain (Muto Pure Chemicals Co., Japan) and differentially counted (300/rat) as alveolar macrophages (AM), polymorphonuclear neutrophils (PMN), and lymphocytes.

The activity of the cytosolic enzyme LDH was used as an indicator of pulmonary damage resulting from general cytotoxicity and was measured by an automatic analyzer (TBA200FR; Toshiba, Japan).

Histopathological evaluation. After the BALF was collected, the right lung was perfused intratracheally with $10 \%$ neutral phosphate-buffered formalin (NPBF) and fixed in $10 \%$ NPBF, pending further processing. Specimens were dehydrated, embedded in paraffin, sectioned as 3- $\mu \mathrm{m}$ slices onto microscope slides, and stained with Masson's Trichrome stain (Sigma-Aldrich, St. Louis, MO). The extent of pulmonary fibrosis was assessed by microscopic examination, as described below. The lesions were histologically graded by two experienced histopathologists using a blinded, scoring system for the extent and severity of inflammation and fibrosis in the lung parenchyma, as previously outlined (Ashcroft et al., 1988).

RNA extraction. Total RNA was extracted from a portion of the left lung sample using Trizol reagent (Invitrogen, Carlsbad, CA) and an RNeasy mini kit (Qiagen, Valencia, CA), according to manufacturers' instructions. Isolated total RNA was quantified using a NanoDrop spectrophotometer (NanoDrop Technologies, Montchanin, DE). For microarray experiments, the quality of RNA was evaluated using a 2100 Bioanalyzer (Agilent Technologies, Palo Alto, CA).

Gene expression analysis. Changes in gene expression in the lungs of bleomycin-treated SD rats were analyzed using an Affymetrix rat genome 2302.0 array. Total RNA samples $(3 \mu \mathrm{g})$ from each control and bleomycin-treated group were used for cDNA synthesis. All microarray procedures, including synthesis of cDNA and biotin-labeled cRNA, fragmentation of cRNA, hybridization, washing, staining, and scanning, were performed according to the manufacturer's instructions. Data normalization, statistical analysis, and selection of differentially expressed genes (DEGs) were performed by GenPlex software, version 3.0 (ISTECH Inc., 
Korea), after raw image data processing by the MARS 5.0 algorithm. The selection of DEGs in the bleomycin-treated groups was based on a greater than 1.5-fold change in expression relative to the corresponding control group. Statistically significant threshold values were assigned at $p<$ 0.05 according to Student's $t$-test. The selected DEGs for days 7,14 , and 28 were combined and analyzed by twodimensional hierarchical clustering and principal components analysis. Classifications of the DEGs by biological function/disease and canonical pathway were performed using Ingenuity Pathway Analysis (IPA) for the data set of significantly altered genes at each time point. The $P$ value, represented as a negative log ratio of the IPA results, is the probability based on Fischer's exact test. In addition, genes that may be involved in bleomycin-induced pulmonary fibrosis were selected using the IPA knowledge base and public databases. The selected genes were annotated based on the NetAffyx ${ }^{\mathrm{TM}}$ and Ingenuity ${ }^{\circledR}$ systems.

Statistical analysis. All measured values are expressed as means \pm standard deviations. Statistical analyses were performed using SAS (Version 4.2.2; Xybion Medical Systems Corp., Cedar Knolls, NJ). Analysis of variance (ANOVA) was used to assess differences between groups, and Student's $t$-test was used for individual comparisons. Statistical significance was established at $p<0.05$ or $p<0.01$.

\section{RESULTS}

BALF analysis. The total and differential cell counts in the BALF of rats at 7, 14, and 28 days after treatment with bleomycin are presented in Table 1 . The numbers of total cells, alveolar macrophages, neutrophils, and lymphocytes were significantly increased at 7 and 14 days compared with the control values $(p<0.01)$. The total number of bronchoalveolar (BAL) cells was highest at 7 days, and the percentage of neutrophils was higher than that in the control group. The total cell number and differential cell counts decreased thereafter. The number of lymphocytes showed a similar increase, but the absolute values were extremely small compared with the numbers of neutrophils and alveolar macrophages.

LDH activity in BALF, which represents the extent of lung cell damage, was significantly higher $(p<0.01)$ at 14 days after bleomycin instillation compared with the control activity (Table 1). The highest LDH activity occurred at day 7.

Histopathological findings. Histopathological changes in the lungs were examined on days 7, 14, and 28 after bleomycin or saline treatment. To confirm the histopathology of bleomycin-induced lung inflammation and fibrosis, the overall histopathological grades of lung inflammation and fibrosis were scored (Table 2). Lung inflammation in the

Table 1. Total and differential cell counts and LDH concentration in BAL fluid

\begin{tabular}{|c|c|c|c|c|c|c|}
\hline \multirow[b]{2}{*}{ Parameter } & \multicolumn{2}{|c|}{ Day 7} & \multicolumn{2}{|c|}{ Day 14} & \multicolumn{2}{|c|}{ Day 28} \\
\hline & Control & Bleomycin & Control & Bleomycin & Control & Bleomycin \\
\hline Total cells $\left(\times 10^{4} / \mathrm{m} l\right)$ & $7.60(1.15)$ & $58.97(11.96)^{* *}$ & $6.28(1.21)$ & $25.08(8.58)^{* *}$ & $9.57(2.11)$ & $15.44(3.62)^{*}$ \\
\hline Macrophages & $7.41(1.09)$ & $27.02(6.67)^{* *}$ & $6.15(1.2)$ & $20.4(6.2)^{* *}$ & $9.36(2.09)$ & $15.00(3.51)^{*}$ \\
\hline Neutrophils & $0.09(0.03)$ & $25.07(6.15)^{* *}$ & $0.05(0.02)$ & $3.49(1.89)^{*}$ & $0.09(0.03)$ & $0.24(0.10)^{*}$ \\
\hline Lymphocytes & $0.09(0.04)$ & $6.88(1.13)^{* *}$ & $0.06(0.02)$ & $1.16(0.64)^{* *}$ & $0.11(0.03)$ & $0.20(0.7)^{*}$ \\
\hline $\mathrm{LDH}(\mathrm{U} / l)$ & $1.2(1.3)$ & $50.2(20.7)^{* *}$ & $4.4(2.88)$ & $11.6(13.46)$ & $0.4(0.5)$ & $0.8(0.04)$ \\
\hline
\end{tabular}

Data are presented as mean \pm S.D of groups.

${ }^{*} p<0.05$ vs. control group.

${ }^{* *} p<0.01$ vs. control group.

Table 2. Incidence and severity of microscopic findings in the lung

\begin{tabular}{lcccc}
\hline \hline \multirow{2}{*}{ Lung pathology } & Control & \multicolumn{3}{c}{ Bleomycin-treated group } \\
\cline { 3 - 5 } & $(\mathrm{n}=5)$ & 7 Day $(\mathrm{n}=5)$ & 14 Day $(\mathrm{n}=5)$ & 28 Day $(\mathrm{n}=5)$ \\
\hline Inflammatory cell infiltration & & $+++++(2),++++(3)$ & $++(3),+(2)$ & $++(2),+(3)$ \\
Pulmonary fibrosis & $+++(2),++(3)$ & $++++(3),+++(2)$ & $+++(3),++(2)$ \\
\hline
\end{tabular}

The number of animal with corresponding lesions was represented in the parentheses.

Severity of lesion was indicated as follows; + , minimal; ++ , mild; +++ , moderate; ++++ , marked; +++++ , severe.

+ , minimal; inflammation ( $10 \%$ of the slide), fibrosis (minimal fibrous thickening of alveolar or bronchiolar walls).

++ , mild; inflammation (10 20\%), fibrosis (mild fibrous thickening of alveolar or bronchiolar walls without obvious damage to the lung architecture).

+++ , moderate; inflammation (20 40\%), fibrosis (increased fibrosis with definite damage to the lung structure and formation of fibrous bands).

++++ , marked; inflammation (40 70\%), fibrosis (severe distortion of the lung structure and large fibrous areas).

+++++ , severe; inflammation ( $>70 \%)$, fibrosis (total fibrous obliteration of the field). 

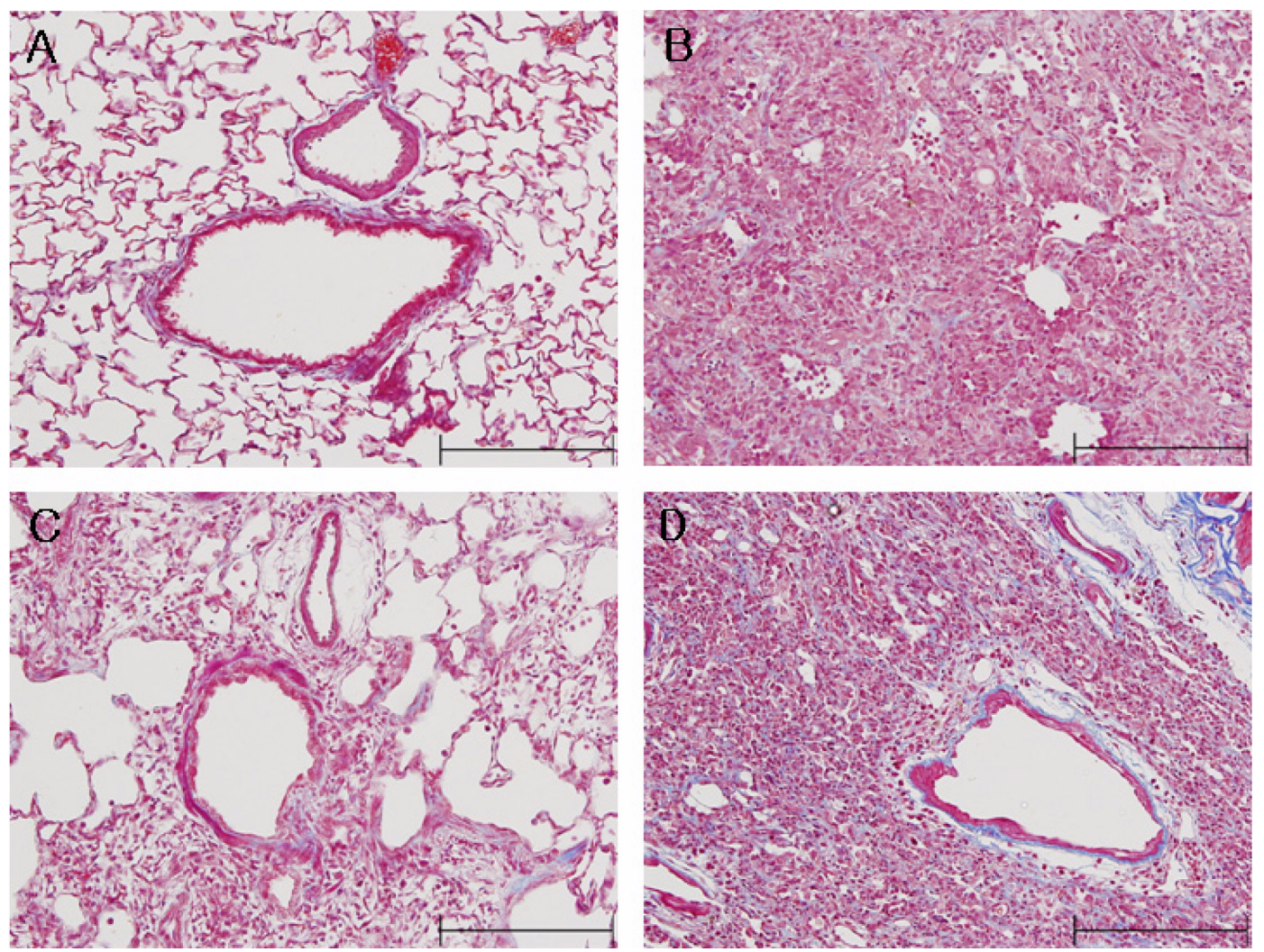

Fig. 1. Representative photomicrographs of lung tissue from bleomycin-instilled rats. A. Control. B. Experimental animal 7 days after ITI of bleomycin. Note the infiltration of numerous neutrophils, alveolar macrophages, and lymphocytes in the peribronchiolar, perivascular, and alveolar spaces, together with thickening of the alveolar interstitium and proliferation of fibroblasts. C. Experimental animal 14 days after ITI of bleomycin. Note the small number of inflammatory cells and numerous fibroblasts, plus a moderate collagenous deposit with infiltration of neutrophils and monocytes. D. Experimental animal 28 days after ITI of bleomycin. Note the moderate alveolar fibrosis and minimal infiltration of inflammatory cells, mainly of neutrophils and few alveolar macrophages. Interstitial thickening is seen in the alveolar walls. Scale bar $=200 \mu \mathrm{m}$.

bleomycin-treated rats peaked at day 7 and decreased thereafter. Representative light photomicrographs of lung tissues from of bleomycin-treated animals are shown in Fig. 1. On day 7 , marked infiltration by inflammatory cells (predominantly macrophages and neutrophils) in the alveolar regions was apparent in the bleomycin-treated rats. Additionally, extensive hyperplasia of alveolar epithelial cells and proliferation of fibroblasts were observed (Fig. 1B). On day 14, the bleomycin-treated rats showed moderate deposition of collagen fibers and fibroblasts in the peribronchiolar and alveolar interstitium, with a few inflammatory cells in the fibrotic area (Fig. 1C). On day 28, diffuse or focal dense fibrosis was observed in the peribronchiolar and subpleural interstitial spaces, with a small number of inflammatory cells (Fig. 1D). The severity of these changes varied among the rats and ranged from mild to moderate.

Gene expression analysis. Compared with the expression patterns in the corresponding controls, differentially expressed genes were detected on days 7, 14, and 28 after bleomycin treatment, based on the fold change and statistical significance ( $\geq 1.5$-fold, $p<0.05$ ). The numbers of significantly altered probes were 2,$839 ; 1,665$; and 1,906 on days 7,14 , and 28 after bleomycin treatment, respectively. We combined all of the selected probe sets, which included 4,662 probe sets that were significantly altered for at least one time point, and analyzed the expression patterns using two-dimensional hierarchical clustering based on the Pearson correlation and complete linkage. Cluster analysis showed distinguishable gene expression patterns between the treatment and control groups and yielded three differential clusters among days 7,14 , and 28 , indicating time dependence (Fig. 2A). An unsupervised principal components analysis revealed four major differences between the controls and the three different time-point groups, further indicating that the gene expression profiles change over time (Fig. 2B). The 10 most significantly up- or down-regulated genes at each time point are presented in Table 3. On days 7 and 14, $\operatorname{Trem} 2, \mathrm{Mmp}$, and Sppl were markedly up-regulated. In contrast, on days 14 and 28, several proteases genes, includ- 
A

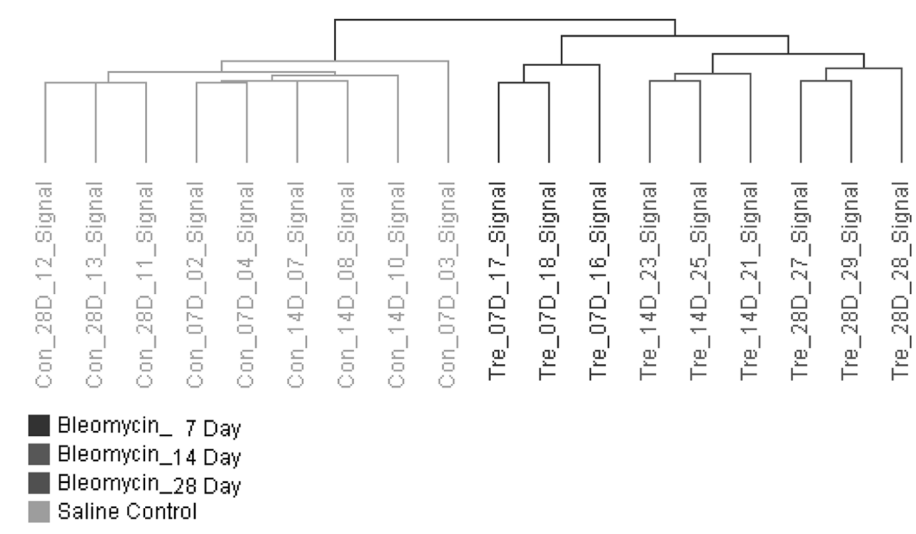

$\mathrm{B}$

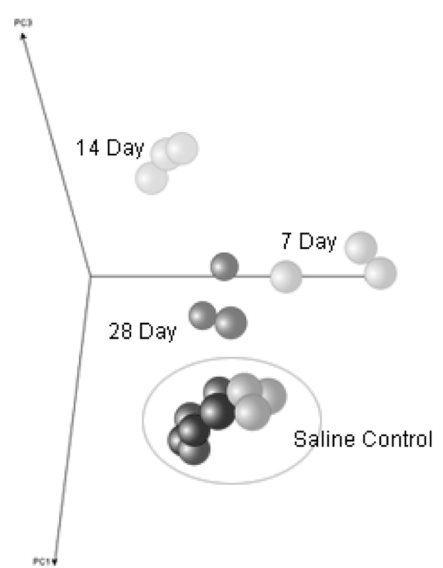

Fig. 2. Hierarchical clustering (A) and principal components analysis (B) using 4,662 selected probe sets.

ing Tpsab1, Mcpt1, and Cma1, were up-regulated. Interestingly, Trem 2 expression was up-regulated by more than 10fold at all time points, and Cyp $2 a 3 a$ was down-regulated by more than 10-fold at all time points.

Classification by function, disease/disorder, and canonical pathway. To understand the overall genomic response to bleomycin, selected probe sets at each time point were imported into the IPA software classification into three categories, namely, biological function, disease/disorder, and canonical pathway, based on the IPA knowledge base. Fig.
3A shows the genes with altered expression at each time point that were classified into the biological function category. The genes significantly altered on day 7 involved mainly cell-to-cell signaling and interactions, antigen presentation, and cellular movement. Fewer genes altered on days 14 and 28 were classified into this category. On day 14 , the main dysregulated genes were those involved in lipid metabolism, small molecule biochemistry, cellular function and maintenance, and cellular growth and proliferation; the expression of genes involved in molecular transport, cell death, and cell signaling gradually increased. The genes

Table 3. Genes significantly modulated in the lungs of bleomycin-treated rats

\begin{tabular}{|c|c|c|c|}
\hline Probe ID & Gene symbol & Gene name & Fold change \\
\hline \multicolumn{4}{|c|}{7 days after bleomycin treatment } \\
\hline \multicolumn{4}{|l|}{ Up-regulated } \\
\hline 1382513_at & Trem 2 predicted & triggering receptor expressed on myeloid cells 2 (predicted) & 82.827 \\
\hline 1368766_at & $\operatorname{Mmp} 7$ & matrix metallopeptidase 7 & 59.790 \\
\hline 1367581_a_at & Sppl & secreted phosphoprotein 1 & 45.831 \\
\hline 1387715 at & Expi & extracellular peptidase inhibitor & 35.944 \\
\hline 1384442_at & Cldn6_predicted & claudin 6 (predicted) & 22.171 \\
\hline 1368587_at & Apocl & apolipoprotein C-I & 18.956 \\
\hline 1393917_at & Cd163 predicted & CD163 antigen (predicted) & 18.320 \\
\hline 1385687_at & LOC682044 & hypothetical protein LOC682044 & 17.710 \\
\hline 1367794_at & $A 2 m$ & alpha-2-macroglobulin & 17.607 \\
\hline 1384329_at & Prss22_predicted & protease, serine, 22 (predicted) & 15.104 \\
\hline \multicolumn{4}{|c|}{ Down-regulated } \\
\hline 1393771_at & Scgb3al & secretoglobin, family $3 \mathrm{~A}$, member 1 & -19.008 \\
\hline 1367985_at & Alas2 & aminolevulinic acid synthase 2 & -17.644 \\
\hline 1387796_at & Alox15 & arachidonate 15-lipoxygenase & -17.603 \\
\hline 1376980_at & $H \operatorname{tr} 2 c$ & 5-hydroxytryptamine (serotonin) receptor $2 \mathrm{C}$ & -16.090 \\
\hline 1368338_at & $C d 52$ & CD52 antigen & -15.683 \\
\hline 1370269_at & Cyplal & cytochrome P450, family 1 , subfamily a, polypeptide 1 & -15.416 \\
\hline 1370355_at & Scdl & stearoyl-Coenzyme A desaturase 1 & -14.370 \\
\hline 1369136 at & Cyp $2 a 3 a$ & cytochrome $\mathrm{P} 450$, family 2 , subfamily A, polypeptide $3 \mathrm{a}$ & -12.412 \\
\hline 1375519_at & LOC287167 & globin, alpha & -12.335 \\
\hline 1385759 at & Serpinb10 & serine (or cysteine) peptidase inhibitor, clade B (ovalbumin), member 10 & -10.382 \\
\hline
\end{tabular}


Table 3. Continued

\begin{tabular}{|c|c|c|c|}
\hline Probe ID & Gene symbol & Gene name & Fold change \\
\hline \multicolumn{4}{|c|}{14 days after bleomycin treatment } \\
\hline \multicolumn{4}{|l|}{ Up-regulated } \\
\hline 1382513_at & Trem 2 predicted & triggering receptor expressed on myeloid cells 2 (predicted) & 65.595 \\
\hline 1387162_at & Tpsabl & tryptase alpha/beta 1 & 64.258 \\
\hline 1370405_at & Mcpt1 & mast cell protease 1 & 46.476 \\
\hline 1368912_at & Trh & thyrotropin releasing hormone & 27.322 \\
\hline 1368766_at & Mmp7 & matrix metallopeptidase 7 & 24.723 \\
\hline 1384442_at & Cldn6_predicted & claudin 6 (predicted) & 20.582 \\
\hline 1387173_at & Cmal & chymase 1 , mast cell & 20.505 \\
\hline 1379331_at & Tnn_predicted & tenascin N (predicted) & 18.944 \\
\hline 1367581_a_at & $\operatorname{Spp} \overrightarrow{1}$ & secreted phosphoprotein 1 & 18.240 \\
\hline 1375052_at & LOC501015 & similar to nuclear localized factor 2 & 13.946 \\
\hline \multicolumn{4}{|c|}{ Down-regulated } \\
\hline 1393771_at & Scgb3al & secretoglobin, family $3 \mathrm{~A}$, member 1 & -53.032 \\
\hline 1369136_at & Cyp $2 a 3 a$ & cytochrome P450, family 2 , subfamily A, polypeptide $3 \mathrm{a}$ & -31.674 \\
\hline 1368608_at & Cyp $2 f 4$ & cytochrome P450, family 2 , subfamily f, polypeptide 4 & -27.077 \\
\hline 1371209_at & $R T 1-C E 5$ & RT1 class I, CE5 & -9.042 \\
\hline 1387839_at & $R T 1-N 1$ & RT1 class Ib gene, H2-TL-like, grc region (N1) & -8.928 \\
\hline 1385759_at & Serpinb10 & serine (or cysteine) peptidase inhibitor, clade B (ovalbumin), member 10 & -8.886 \\
\hline 1373368_at & LOC684050 & similar to procollagen C-endopeptidase enhancer 2 & -6.774 \\
\hline 1375842_at & LOC688972 & similar to Glycophorin & -6.175 \\
\hline 1370789_a_at & Prlr & prolactin receptor & -5.001 \\
\hline 1369435_-̄at & Ttpa & tocopherol (alpha) transfer protein & -4.651 \\
\hline \multicolumn{4}{|c|}{28 days after bleomycin treatment } \\
\hline \multicolumn{4}{|l|}{ Up-regulated } \\
\hline 1370405_at & Mcpt1 & mast cell protease 1 & 48.603 \\
\hline 1387162_at & Tpsabl & tryptase alpha/beta 1 & 44.757 \\
\hline 1387173_at & Cmal & chymase 1 , mast cell & 30.695 \\
\hline 1382513_at & Trem 2 predicted & triggering receptor expressed on myeloid cells 2 (predicted) & 26.319 \\
\hline 1396388_at & LOC691835 & similar to Potassium channel tetramerisation domain-containing protein 4 & 20.559 \\
\hline 1370971_at & Myhl & myosin, heavy polypeptide 1 , skeletal muscle, adult & 18.842 \\
\hline 1374863_at & RGD1562168_predicted & similar to retinoid binding protein 7 (predicted) & 17.563 \\
\hline 1371164_at & Mcpt10 & mast cell protease 10 & 15.911 \\
\hline 1373865_at & Snap 91 & synaptosomal-associated protein, $91 \mathrm{kDa}$ homolog (mouse) & 15.259 \\
\hline 1368519_at & Serpine1 & serine (or cysteine) peptidase inhibitor, clade E, member 1 & 14.869 \\
\hline \multicolumn{4}{|c|}{ Down-regulated } \\
\hline 1380363_at & Klf7_predicted & Kruppel-like factor 7 (ubiquitous) (predicted) & -15.640 \\
\hline 1369136_at & Cyp $\overrightarrow{2 a} 3 a$ & cytochrome P450, family 2 , subfamily A, polypeptide $3 \mathrm{a}$ & -11.061 \\
\hline 1368608_at & Cyp $2 f 4$ & cytochrome $\mathrm{P} 450$, family 2 , subfamily f, polypeptide 4 & -9.198 \\
\hline 1375148_at & LOC691504 & similar to Zinc finger protein ZFPM1 (Zinc finger protein multitype 1) & -8.712 \\
\hline 1369300_at & Nor 1 & natural cytotoxicity triggering receptor 1 & -8.316 \\
\hline 1378089_at & $M G C 108778$ & Similar to RIKEN cDNA $1810057 \mathrm{C} 19$ & -7.732 \\
\hline 1391864_at & Enpp6_predicted & Ectonucleotide pyrophosphatase/phosphodiesterase 6 (predicted) & -6.857 \\
\hline 1380799_at & Grem 2 predicted & gremlin 2 homolog, cysteine knot superfamily (predicted) & -6.740 \\
\hline 1387631_at & Hpgd & hydroxyprostaglandin dehydrogenase 15 (NAD) & -6.074 \\
\hline 1369703_at & Epas 1 & endothelial PAS domain protein 1 & -5.876 \\
\hline
\end{tabular}

classified into 11 disease/disorder-associated groups are presented in Fig. 3B. Respiratory disease comprised the highest number of altered genes, and many dysregulated genes associated with inflammatory diseases and genetic disorders were identified on day 7 . The majority of genes with altered expression on day 28 were related to inflammatory diseases and genetic disorders, although there were also a high number associated with skeletal/muscular and connective tissue disorders. We also examined genes of canonical pathways associated with cytokine signaling and cell-mediated immune responses (Fig. 4). Most of the genes for cytokines, including TREM, IL-10, IL-4, IL-8, and IL-6, were highly dysregulated on day 7 after bleomycin treatment. 

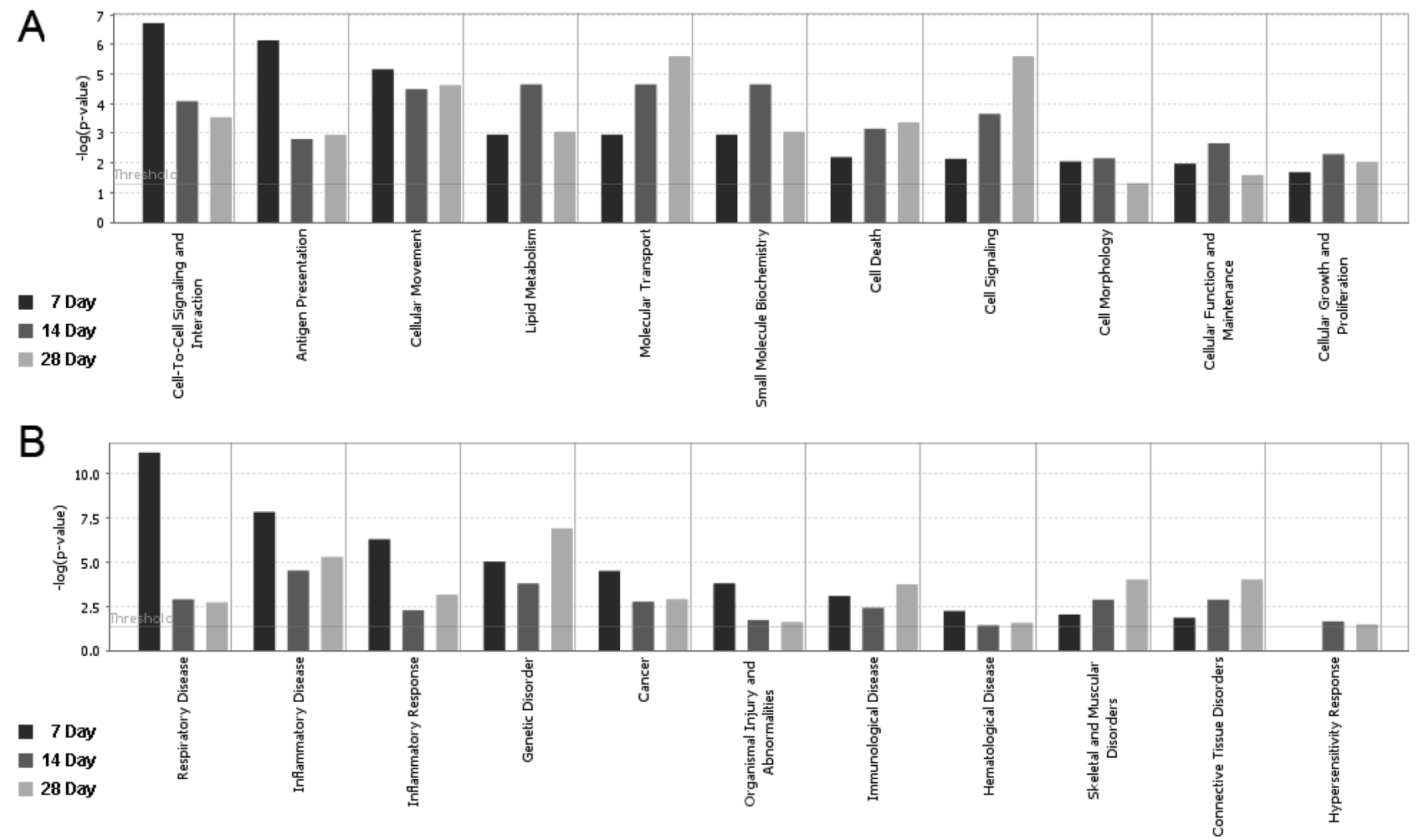

Fig. 3. Functions and diseases affected as a result of altered gene expression in the lungs of belomycin-treated rats. A. Top functions. B. Top diseases and disorders. Bars represent $-\log (p$-value) for the disproportionate representation of the affected genes among the total number of genes in the selected function/disease category. Yellow line represents the threshold $(p=0.05)$ level.

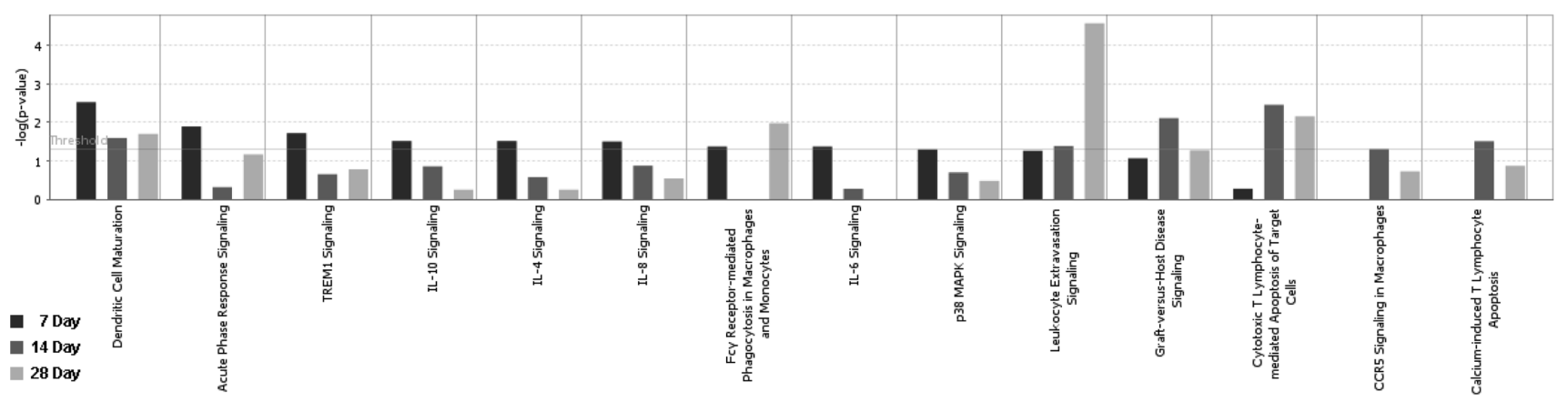

Fig. 4. Analysis of canonical pathways associated with cytokine signaling and cell-mediated immune response in the lungs of bleomycin-treated rats. Bars represent -log(p-value) for the disproportionate representation of affected genes among the total number of genes in the selected function/disease category. Yellow line represents the threshold $(p=0.05)$ level.

Altered gene expression involved in the development of fibrosis. Bleomycin-induced pulmonary fibrosis in the rodent is a well-established model for histopathological, biochemical, and molecular biology studies, and numerous studies have revealed the regulation of genes associated with pulmonary fibrosis in bleomycin-treated, transgenic, and knock-out mice. To compare our gene expression data with the results of previous studies, we extracted 52 genes associated with common fibrogenesis mechanisms and ble- omycin-induced pulmonary fibrosis, using the IPA knowledge base and public databases. As listed in Table 4, the expression profiles of the following genes were significantly modulated: fibrogenic cytokines and cytokine receptors such as Il-6, Il-13ral, Il-13ra2, Tgfb1, and Tgfb2; fibrogenic chemokines and chemokine receptors such as $\mathrm{Ccl}$ 2, Ccl3, Ccrl, and Ccr2; growth factors such as Egrl and Ctgf; extracellular matrix components such as Actg2, Eln, Fn1, Collal, and Colla3; and several proteases, 
Table 4. Modulation of fibrosis-related genes in the lungs of bleomycin-treated rats

\begin{tabular}{|c|c|c|c|c|c|}
\hline \multirow{2}{*}{ Probe set ID } & \multirow{2}{*}{ Gene symbol } & \multirow{2}{*}{ Gene name } & \multicolumn{3}{|c|}{ Fold change } \\
\hline & & & 7 day & 14 day & 28 day \\
\hline 1386869_at & Actg2 & actin, gamma 2 , smooth muscle, enteric & 2.885 & 1.736 & 1.731 \\
\hline 1374207_at & Angpt2 & angiopoietin 2 & 1.319 & 1.073 & 1.522 \\
\hline 1369122_at & $B a x$ & Bcl2-associated $\mathrm{X}$ protein & 1.959 & 1.600 & 1.115 \\
\hline 1377759_at & Bid & $\mathrm{BH} 3$ interacting domain death agonist & 2.188 & 1.294 & 1.131 \\
\hline 1382783_at & Blm & Bloom syndrome homolog (human) & 1.807 & 1.187 & 1.253 \\
\hline 1390386_at & Casp3 & caspase 3 , apoptosis related cysteine protease & 1.938 & 1.360 & 1.031 \\
\hline 1370131_at & Cavl & caveolin, caveolae protein 1 & -2.469 & -1.349 & -4.636 \\
\hline 1370118_at & $\operatorname{Ccl17}$ & chemokine (C-C motif) ligand 17 & 6.033 & 2.922 & 2.542 \\
\hline 1367973_at & $\mathrm{Ccl} 2$ & chemokine (C-C motif) ligand 2 & 7.651 & 7.096 & 4.366 \\
\hline 1370620_at & Ccl22 & chemokine (C-C motif) ligand 22 & 8.574 & 2.879 & 3.207 \\
\hline 1369815_at & $\mathrm{Ccl} 3$ & chemokine (C-C motif) ligand 3 & 1.421 & 1.632 & 2.391 \\
\hline 1369983_at & Ccl5 & chemokine (C-C motif) ligand 5 & -1.232 & -3.072 & -2.507 \\
\hline 1370083_at & Ccrl 1 & chemokine (C-C motif) receptor 1 & 1.295 & 2.001 & 1.630 \\
\hline 1387742_at & Ccr2 & chemokine (C-C motif) receptor 2 & 3.201 & 1.777 & -3.926 \\
\hline 1370034_at & $C d c 25 b$ & cell division cycle 25 homolog B (S. pombe) & -3.997 & -2.057 & -1.177 \\
\hline 1387391_at & Cdknla & cyclin-dependent kinase inhibitor $1 \mathrm{~A}(\mathrm{p} 21, \mathrm{Cip} 1)$ & 6.769 & 2.970 & 2.014 \\
\hline 1370864_at & Collal & collagen, type I, alpha 1 & 1.792 & 2.920 & 3.944 \\
\hline 1370959_at & Col3al & collagen, type III, alpha 1 & 1.102 & 1.270 & 1.702 \\
\hline 1367631_at & $\operatorname{Ctg} f$ & connective tissue growth factor & 1.469 & 1.804 & 1.657 \\
\hline 1369947_at & Ctsk & cathepsin $\mathrm{K}$ & 6.666 & 7.213 & 7.603 \\
\hline 1387316 at & Cxcll & chemokine (C-X-C motif) ligand 1 & 1.390 & -1.298 & -1.975 \\
\hline 1368760_at & Cxcl2 & chemokine (C-X-C motif) ligand 2 & 2.777 & 1.357 & -1.076 \\
\hline 1370633_at & Cxcl3 & chemokine (C-X-C motif) ligand 3 & 2.261 & 1.699 & -1.547 \\
\hline $1370699^{-}$a at & Egfr /// Pepd & epidermal growth factor receptor /// peptidase D & 2.272 & 1.739 & 1.316 \\
\hline 1368321_at & Egrl & early growth response 1 & 1.950 & 1.337 & 1.748 \\
\hline 1388111_at & Eln & elastin & 1.168 & 1.883 & 2.628 \\
\hline 1370234_at & Fnl & fibronectin 1 & 1.967 & 1.957 & 1.923 \\
\hline 1387386_at & Foxj1 & forkhead box J1 & 1.212 & -2.564 & -1.135 \\
\hline 1371727_at & Glb1 & galactosidase, beta 1 & 1.788 & 1.506 & 1.606 \\
\hline 1370331_at & Ill1ral & interleukin 11 receptor, alpha chain 1 & -1.558 & -1.137 & 1.138 \\
\hline 1370728_at & Il13ral & interleukin 13 receptor, alpha 1 & 1.561 & 1.373 & -1.141 \\
\hline 1369266_at & Ill $13 r a 2$ & interleukin 13 receptor, alpha 2 & 2.148 & 1.786 & 2.544 \\
\hline 1369191_at & Il6 & interleukin 6 & 5.899 & 3.800 & 3.031 \\
\hline 1387144_at & Itgal & integrin alpha 1 & 1.315 & -2.246 & -5.186 \\
\hline 1383880_at & Itgav_predicted & integrin alpha $\mathrm{V}$ (predicted) & 2.941 & 2.340 & 1.613 \\
\hline 1386879_at & Lgals3 & lectin, galactose binding, soluble 3 & 1.790 & 1.558 & 1.291 \\
\hline 1387946_at & Lgals $3 b p$ & lectin, galactoside-binding, soluble, 3 binding protein & 2.029 & 1.532 & 1.382 \\
\hline 1379612_at & Mapk8 & mitogen-activated protein kinase 8 & 1.507 & 1.095 & 1.109 \\
\hline 1384427_at & $M d m 2$ & Mdm2 p53 binding protein homolog (mouse) & 2.762 & 1.304 & -1.694 \\
\hline 1368530_at & Mmpl2 & matrix metallopeptidase 12 & 6.566 & 7.207 & 7.116 \\
\hline 1370301_at & Mmp2 & matrix metallopeptidase 2 & 1.928 & 1.359 & 1.738 \\
\hline 1369200_at & Nt5e & 5' nucleotidase, ecto & 1.422 & 1.185 & 1.933 \\
\hline 1385434_at & Ptger 2 & Prostaglandin E receptor 2, subtype EP2 & 2.695 & 1.414 & 1.676 \\
\hline 1367780_at & Pttg1 & pituitary tumor-transforming 1 & 1.771 & 1.650 & 1.886 \\
\hline 1368423_at & Retnla & resistin like alpha & 3.994 & 2.069 & 2.216 \\
\hline 1368519_at & Serpinel & serine peptidase inhibitor, clade $\mathrm{E}$, member 1 & 6.874 & 3.257 & 14.869 \\
\hline 1367581_a_at & Sppl & secreted phosphoprotein 1 & 45.831 & 18.240 & 8.895 \\
\hline 1370082_at & $T g f b 1$ & transforming growth factor, beta 1 & 2.130 & 1.287 & 1.176 \\
\hline 1376425_at & $\operatorname{Tg} f b 2$ & Transforming growth factor, beta 2 & 1.104 & 2.117 & 1.004 \\
\hline 1367712_at & Timpl & TIMP metallopeptidase inhibitor 1 & 6.731 & 2.685 & 1.692 \\
\hline 1370752_a_at & Tp53 & tumor protein $\mathrm{p} 53$ & 1.550 & 1.599 & 1.505 \\
\hline 1389234_at & $V w f$ & Von Willebrand factor homolog & -1.859 & -1.317 & -1.216 \\
\hline
\end{tabular}

Bold; $p<0.05$ compared with control group. 
including Ctsk, Mmp2, and Mmp12.

\section{DISCUSSION}

In the present study, we confirmed bleomycin-induced pulmonary fibrosis in a SD rat model using histopathological examination and BALF analysis. Consistent with previous findings, the histopathological results showed markedly increased inflammation, with infiltrated immune cells extending into the alveolar regions and proliferation of epithelial cells and fibroblasts at day 7; moderate deposition of collagen at day 14; and dense fibrosis in the peribronchiolar and interstitial spaces with reduced inflammation foci at day 28. LDH activity was highest on day 7 after bleomycin treatment. Based on these results, we investigated the gene expression profiles associated with fibrogenic mechanisms in the lungs of this rat model, using well-documented findings and the IPA knowledge base for comparisons.

During the development of fibrosis, IL-4, IL-5, and IL-13 secreted by $\mathrm{CD}^{+} \mathrm{T}$ helper type 2 cells $\left(\mathrm{T}_{\mathrm{H}} 2 \mathrm{~s}\right)$ play important roles in several cytokine-deficient mice (Wynn and Sher, 1995). Early microarray-based gene expression profiling revealed that $\mathrm{T}_{\mathrm{H}} 1$ and $\mathrm{T}_{\mathrm{H}} 2$ cytokines induced markedly different genomic responses to pathogen-induced inflammation in mice, and differences in the induction of several genes associated with fibrogenic mechanisms have been shown in $\mathrm{T}_{\mathrm{H}} 2$ polarized mice (Hoffmann et al., 2001; Sandler et al., 2003). In our study, direct gene induction of $I l-4$, $I l-5$, and $I l-13$, which are the primary $\mathrm{T}_{\mathrm{H}} 2$ cytokines, was not observed; however, the expression of genes related to the IL-4 signaling pathway was significantly altered on day 7 after bleomycin treatment (Fig. 4). IL-4 is a profibrotic cytokine that directly stimulates the in vitro synthesis of extracellular matrix (ECM) proteins, including type I and type III collagen, and fibronectin (Fertin et al., 1991; Doucet et al., 1998). Our data demonstrate significant induction of genes encoding collagen type I- $\alpha 1$ (Collal) and type III- $\alpha 1$ (Col3al) and fibronectin, consistent with previous findings.

Among the $\mathrm{T}_{\mathrm{H}} 2$ cytokines, IL-13 is one of the most powerful fibrogenic cytokines. Selected expression of IL-13 in the lungs of mice caused severe subepithelial fibrosis (Zhu et al., 1999). Furthermore, antibody-mediated inhibition of IL-13 attenuated collagen deposition in mice with bleomycininduced pulmonary fibrosis (Belperio et al., 2001). Although the induction of $I l-13$ was not detected in our study, we did find the induction of IL-13 receptor genes such as Il-13ral and $\mathrm{Il}-13 \mathrm{ra}$. IL-13R $\alpha 2$ is an inhibitory decoy receptor of IL-13 and thereby attenuates the progression of fibrosis; it has been considered to serve mainly as a negative-feedback mechanism for the $\mathrm{T}_{\mathrm{H}} 2$ response (Wynn, 2004). However, a recent study has demonstrated that IL-13 signaling through IL-13R $\alpha 2$ is involved in the induction of TGF- $\beta 1$ production and collagen deposition (Fichtner et al., 2006). Moreover, increases in IL-13R $\alpha 1$ and IL-13R $\alpha 2$ in cultured fibroblast cells from an idiopathic interstitial pneumonia patient have been reported (Jakubzick et al., 2004).

In addition to the induction of genes related to $\mathrm{T}_{\mathrm{H}} 2$ cytokines, $T g f b 1$ and $T g f b 2$ were significantly up-regulated on days 7 and 14, respectively. TGF- $\beta$ is a major regulator of the ECM, and the induction of TGF- $\beta$ is tightly associated with the development of fibrosis (Kaminski et al., 2000; Sime et al., 1997). Of the three TGF- $\beta$ isoforms, TGF- $\beta 1$ is the main ECM regulator and is stored in the cell in an inactive form, bound by latency-associated protein (LAP). For TGF- $\beta 1$ activation, the LAP-TGF- $\beta 1$ complex must be cleaved through a process catalyzed by integrin- $\alpha_{v} \beta_{6}$ or proteolytic enzymes such as cathepsins or matrix metalloproteinases (MMPs) (Munger et al., 1999; Gorelik and Flavell, 2002). In the present study, two genes linked with the dissociation of the LAP-TGF- $\beta 1$ complex, i.e., Mmp 2 and integrin- $\alpha_{v}$ (Itgav), were up-regulated in the lungs of SD rats after bleomycin treatment. Activated TGF- $\beta$ modulates fibrogenic effects through SMAD protein, which triggers the transcription of target genes such as Collal, Colla3, and connective tissue growth factor (Ctgf) (Roberts et al., 2003; Perbal, 2004). Although an elevated SMAD expression level was not detected, the induction of Ctgf, Collala, and Colla3, which are downstream of the TGF- $\beta 1-S M A D 3$ signaling pathway, were observed. Ctgf is an important fibrogenic modulator and regulates fibroblast proliferation, cellular adhesion, chemotaxis, and ECM deposition (Perbal, 2004). On the other hand, it has been reported that TGF- $\beta 1$ stimulates pulmonary fibrosis via $\mathrm{Bcl} 2$-associated $\mathrm{X}$ protein (Bax), BH3 interacting domain death agonist (Bid), and MMP12 (Kang et al., 2007). TGF- $\beta 1$ is an in vivo stimulator of Bax, Bid, Timp-1, and Mmp-12, and the Bid- and Bax-mediated apoptosis pathways are important in the pathogenesis of pulmonary inflammation and fibrosis. In addition, Mmp12, which is downstream of TGF- $\beta 1$ via the Bid- and Baxmediated pathways, is a main contributor to the pathogenesis of TGF- $\beta 1$ - and bleomycin-induced pulmonary fibrosis (Kang et al., 2007). Elevated expression levels of Bid, Bax, and Timp- 1 were observed on day 7 in our study, and significantly increased $M m p 12$ expression levels were maintained up to day 28 after bleomycin treatment.

The CC-chemokine family is known to be strongly involved in the development of fibrosis via cooperation with profibrotic cytokines such as IL-13 and TGF- $\beta$ (Ma et al., 2004; Murray et al., 2008). In our study, several CC-chemokine genes, including $\mathrm{Ccl} 2, \mathrm{Ccl} 3, \mathrm{Ccl} 17 \beta$ and $\mathrm{Ccl} 22$, and $\mathrm{CC}$ chemokine receptor genes, including $C c r 1$ and $C c r 2$, were significantly up-regulated. In particular, CCL2 and CCL3 have been identified as essential mediators of fibrosis, and several CCL2/CCL3 inhibition studies have shown marked reduction of fibrosis in murine models (Smith et al., 1995; Lloyd et al., 1997). Furthermore, findings from studies using CCR1- and CCR2-deficient mice have confirmed the importance of the CCL2/CCL3 signaling pathway in fibrosis 
development (Tokuda et al., 2000; Moore et al., 2001).

Taken together with the induction of well-known genes associated with pulmonary fibrogenesis, our data show that several genes such as triggering receptor expressed on myeloid cells 2 (Trem 2), Mmp 7, Mmp 12, cathepsin K (Ctsk), and secreted phosphoprotein 1 (Spp 1) are significantly increased more than 6-fold and that these expression levels persist up to 28 days after bleomycin treatment. Among the selected genes, Trem 2 showed the highest induction on days 7 and 14 after bleomycin treatment. The molecular function of Trem 2 is not well documented; however, a previous study reported high and persistent induction of Trem 2 expression in stainless steel welding hume-induced lung injury in SD rats (Oh et al., 2009). Sppl is a key molecule in the development of angiotensin II-induced cardiac fibrosis and remodeling (Matsui et al., 2004). Recent studies have shown that Sppl has a pivotal role in smoking-related interstitial lung diseases, which are characterized by the accumulation of macrophages and Langerhans cells, and in fibrotic remodeling (Prasse et al., 2009). The overexpression of Ctsk in silica-induced pulmonary fibrosis in mice has also been reported (van den Brule et al., 2005). In addition, gene expression levels of some proteases such as tryptase alpha/ beta 1 (Tpsab1), mast cell protease 1 ( Mcpt 1$)$, and chymase 1 mast cell ( $\mathrm{Cmal})$ were markedly increased on day 14 after bleomycin treatment. Although these proteases have not been reported to be associated with pulmonary fibrosis, the remarkable induction of these genes with to the emergence of fibroblasts suggests that they may contribute to the development of fibrosis via degradation of the ECM.

In summary, we have investigated changes in gene expression associated with the development of bleomycin-induced pulmonary fibrosis in SD rats. The genes found to be significantly modulated during the development of pulmonary fibrosis correlated well with the histopathological changes and previously published findings. Our data will aid in an understanding of the underlying mechanisms involved in the development of fibrosis and will contribute to efforts aimed at identifying candidate biomarkers for fibrotic disease.

\section{ACKNOWLEDGMENTS}

This work was supported by the Ministry of Knowledge Economy with a 2009 General Project grant through the International Inhalation Toxicology Evaluation Technology program at the Korea Institute of Toxicology.

\section{REFERENCES}

Ashcroft, T., Simpson, J.M. and Timbrell, V. (1988). Simple method of estimating severity of pulmonary fibrosis on a numerical scale. J. Clin. Pathol., 41, 467-470.

Belperio, J.A., Keane, M.P., Burdick, M.D., Lynch, J.P., 3rd, Xue, Y.Y., Berlin, A., Ross, D.J., Kunkel, S.L., Charo, I.F. and Stri- eter, R.M. (2001). Critical role for the chemokine MCP-1/ CCR2 in the pathogenesis of bronchiolitis obliterans syndrome. J. Clin. Invest., 108, 547-556.

Cortijo, J., Cerda-Nicolas, M., Serrano, A., Bioque, G., Estrela, J.M., Santangelo, F., Esteras, A., Llombart-Bosch, A. and Morcillo, E.J. (2001). Attenuation by oral N-acetylcysteine of bleomycin-induced lung injury in rats. Eur. Respir. J., 17, 12281235.

Doucet, C., Brouty-Boye, D., Pottin-Clemenceau, C., Canonica, G.W., Jasmin, C. and Azzarone, B. (1998). Interleukin (IL) 4 and IL-13 act on human lung fibroblasts. Implication in asthma. J. Clin. Invest., 101, 2129-2139.

Fertin, C., Nicolas, J.F., Gillery, P., Kalis, B., Banchereau, J. and Maquart, F.X. (1991). Interleukin-4 stimulates collagen synthesis by normal and scleroderma fibroblasts in dermal equivalents. Cell. Mol. Biol., 37, 823-829.

Fichtner-Feigl, S., Strober, W., Kawakami, K., Puri, R.K. and Kitani, A. (2006). IL-13 signaling through the IL-13alpha2 receptor is involved in induction of TGF-beta1 production and fibrosis. Nat. Med., 12, 99-106.

Gharaee-Kermani, M., Hu, B., Phan, S.H. and Gyetko, M.R. (2008). The role of urokinase in idiopathic pulmonary fibrosis and implication for therapy. Expert. Opin. Investig. Drugs, 17, 905-916.

Gharaee-Kermani, M. and Phan, S.H. (2005). Molecular mechanisms of and possible treatment strategies for idiopathic pulmonary fibrosis. Curr. Pharm. Des., 11, 3943-3971.

Gharaee-Kermani, M., Ullenbruch, M. and Phan, S.H. (2005). Animal models of pulmonary fibrosis. Methods Mol. Med., 117, 251-259.

Gorelik, L. and Flavell, R.A. (2002). Transforming growth factorbeta in T-cell biology. Nat. Rev. Immunol., 2, 46-53.

Hoffmann, K.F., McCarty, T.C., Segal, D.H., Chiaramonte, M., Hesse, M., Davis, E.M., Cheever, A.W., Meltzer, P.S., Morse, H.C., 3rd and Wynn, T.A. (2001). Disease fingerprinting with cDNA microarrays reveals distinct gene expression profiles in lethal type 1 and type 2 cytokine-mediated inflammatory reactions. Faseb. J., 15, 2545-2547.

Jakubzick, C., Choi, E.S., Carpenter, K.J., Kunkel, S.L., Evanoff, H., Martinez, F.J., Flaherty, K.R., Toews, G.B., Colby, T.V., Travis, W.D., Joshi, B.H., Puri, R.K., and Hogaboam, C.M. (2004). Human pulmonary fibroblasts exhibit altered interleukin-4 and interleukin-13 receptor subunit expression in idiopathic interstitial pneumonia. Am. J. Pathol., 164, 1989-2001.

Jordana, M., Dolovich, M., Irving, L.B., Tomioka, M., Befus, D., Gauldie, J. and Newhouse, M.T. (1988). Solute movement across the alveolar-capillary membrane after intratracheally administered bleomycin in rats. Am. Rev. Respir. Dis., 138, 96100.

Kaminski, N., Allard, J.D., Pittet, J.F., Zuo, F., Griffiths, M.J., Morris, D., Huang, X., Sheppard, D. and Heller, R.A. (2000). Global analysis of gene expression in pulmonary fibrosis reveals distinct programs regulating lung inflammation and fibrosis. Proc. Natl. Acad. Sci. USA, 97, 1778-1783.

Kang, H.R., Cho, S.J., Lee, C.G., Homer, R.J. and Elias, J.A. (2007). Transforming growth factor (TGF)-betal stimulates pulmonary fibrosis and inflammation via a Bax-dependent, bidactivated pathway that involves matrix metalloproteinase-12. $J$. Biol. Chem., 282, 7723-7732. 
Katsuma, S., Nishi, K., Tanigawara, K., Ikawa, H., Shiojima, S., Takagaki, K., Kaminishi, Y., Suzuki, Y., Hirasawa, A., Yano J., Murakami, Y. and Tsujimoto G. (2001). Molecular monitoring of bleomycin-induced pulmonary fibrosis by cDNA microarray-based gene expression profiling. Biochem. Biophys. Res. Commun., 288, 747-751.

Kuwano, K., Hagimoto, N. and Hara, N. (2001). Molecular mechanisms of pulmonary fibrosis and current treatment. Curr. Mol. Med., 1,551-573.

Lloyd, C.M., Minto, A.W., Dorf, M.E., Proudfoot, A., Wells, T.N., Salant, D.J. and Gutierrez-Ramos, J.C. (1997). RANTES and monocyte chemoattractant protein-1 (MCP-1) play an important role in the inflammatory phase of crescentic nephritis, but only MCP-1 is involved in crescent formation and interstitial fibrosis. J. Exp. Med., 185, 1371-1380.

Ma, B., Zhu, Z., Homer, R.J., Gerard, C., Strieter, R. and Elias, J.A. (2004). The C10/CCL6 chemokine and CCR1 play critical roles in the pathogenesis of IL-13-induced inflammation and remodeling. J. Immunol., 172, 1872-1881.

Matsui, Y., Jia, N., Okamoto, H., Kon, S., Onozuka, H., Akino, M., Liu, L., Morimoto, J., Rittling, S.R., Denhardt, D., Kitabatake, A. and Uede, T. (2004). Role of osteopontin in cardiac fibrosis and remodeling in angiotensin II-induced cardiac hypertrophy. Hypertension, 43, 1195-1201.

Moeller, A., Ask, K., Warburton, D., Gauldie, J. and Kolb, M. (2008). The bleomycin animal model: a useful tool to investigate treatment options for idiopathic pulmonary fibrosis? Int. $J$. Biochem. Cell Biol., 40, 362-382.

Moore, B.B., Paine, R., 3rd, Christensen, P.J., Moore, T.A., Sitterding, S., Ngan, R., Wilke, C.A., Kuziel, W.A. and Toews, G.B. (2001). Protection from pulmonary fibrosis in the absence of CCR2 signaling. J. Immunol., 167, 4368-4377.

Munger, J.S., Huang, X., Kawakatsu, H., Griffiths, M.J., Dalton, S.L., Wu, J., Pittet, J.F., Kaminski, N., Garat, C., Matthay, M.A., Rifkin, D.B. and Sheppard, D. (1999). The integrin alpha v beta 6 binds and activates latent TGF beta 1: a mechanism for regulating pulmonary inflammation and fibrosis. Cell, 96, 319-328.

Murray, L.A., Argentieri, R.L., Farrell, F.X., Bracht, M., Sheng, H., Whitaker, B., Beck, H., Tsui, P., Cochlin, K., Evanoff, H.L., Hogaboam, C.M. and Das, A.M. (2008). Hyper-responsiveness of IPF/UIP fibroblasts: interplay between TGFbeta1, IL-13 and CCL2. Int. J. Biochem. Cell. Biol., 40, 2174-2182.

Oh, J.H., Yang, M.J., Yang, Y.S., Park, H.J., Heo, S.H., Lee, E.H., Song, C.W. and Yoon, S. (2009). Microarray-based analysis of the lung recovery process after stainless-steel welding fume exposure in Sprague-Dawley rats. Inhal. Toxicol., 21, 347-373.

Perbal, B. (2004). CCN proteins: multifunctional signalling regulators. Lancet, 363, 62-64.

Piguet, P.F., Collart, M.A., Grau, G.E., Sappino, A.P. and Vassalli, P. (1990). Requirement of tumour necrosis factor for development of silica-induced pulmonary fibrosis. Nature, 344, 245-247.

Pottier, N., Chupin, C., Defamie, V., Cardinaud, B., Sutherland, R., Rios, G., Gauthier, F., Wolters, P.J., Berthiaume, Y., Barbry, P. and Mari, B. (2007). Relationships between early inflammatory response to bleomycin and sensitivity to lung fibrosis: a role for dipeptidyl-peptidase I and tissue inhibitor of metalloproteinase3? Am. J. Respir. Crit. Care. Med., 176, 1098-1107.

Prasse, A., Stahl, M., Schulz, G., Kayser, G., Wang, L., Ask, K., Yalcintepe, J., Kirschbaum, A., Bargagli, E., Zissel, G., Kolb,
M., Muller-Quernheim, J., Weiss, J.M. and Renkl, A.C. 2009. Essential role of osteopontin in smoking-related interstitial lung diseases. Am. J. Pathol., 174, 1683-1691.

Roberts, A.B., Russo, A., Felici, A. and Flanders, K.C. (2003). Smad3: a key player in pathogenetic mechanisms dependent on TGF-beta. Ann. N. Y. Acad. Sci., 995, 1-10.

Sakanashi, Y., Takeya, M., Yoshimura, T., Feng, L., Morioka, T. and Takahashi, K. (1994). Kinetics of macrophage subpopulations and expression of monocyte chemoattractant protein-1 (MCP-1) in bleomycin-induced lung injury of rats studied by a novel monoclonal antibody against rat MCP-1. J. Leukoc. Biol., 56, 741-750.

Sandler, N.G., Mentink-Kane, M.M., Cheever, A.W. and Wynn, T.A. (2003). Global gene expression profiles during acute pathogen-induced pulmonary inflammation reveal divergent roles for Th1 and Th2 responses in tissue repair. J. Immunol., 171, 3655-3667.

Selman, M., Thannickal, V.J., Pardo, A., Zisman, D.A., Martinez, F.J. and Lynch, J.P., 3rd. (2004). Idiopathic pulmonary fibrosis: pathogenesis and therapeutic approaches. Drugs, 64, 405-430.

Sime, P.J., Xing, Z., Graham, F.L., Csaky, K.G. and Gauldie, J. (1997). Adenovector-mediated gene transfer of active transforming growth factor-beta1 induces prolonged severe fibrosis in rat lung. J. Clin. Invest., 100, 768-776.

Smith, R.E., Strieter, R.M., Zhang, K., Phan, S.H., Standiford, T.J., Lukacs, N.W. and Kunkel, S.L. (1995). A role for C-C chemokines in fibrotic lung disease. J. Leukoc. Biol., 57, 782-787.

Tokuda, A., Itakura, M., Onai, N., Kimura, H., Kuriyama, T. and Matsushima, K. (2000). Pivotal role of CCR1-positive leukocytes in bleomycin-induced lung fibrosis in mice. J. Immunol., 164, 2745-2751.

van den Brule, S., Misson, P., Buhling, F., Lison, D. and Huaux, F. (2005). Overexpression of cathepsin K during silica-induced lung fibrosis and control by TGF-beta. Respir. Res., 6, 84 .

Wynn, T.A. (2004). Fibrotic disease and the T(H)1/T(H)2 paradigm. Nat. Rev. Immunol., 4, 583-594.

Wynn, T.A., Cheever, A.W., Jankovic, D., Poindexter, R.W., Caspar, P., Lewis, F.A. and Sher, A. (1995). An IL-12-based vaccination method for preventing fibrosis induced by schistosome infection. Nature, 376, 594-596.

Zhang, H.Y., Gharaee-Kermani, M., Zhang, K., Karmiol, S. and Phan, S.H. (1996). Lung fibroblast alpha-smooth muscle actin expression and contractile phenotype in bleomycin-induced pulmonary fibrosis. Am. J. Pathol., 148, 527-37.

Zhang, K., Gharaee-Kermani, M., Jones, M.L., Warren, J.S. and Phan, S.H. (1994). Lung monocyte chemoattractant protein-1 gene expression in bleomycin-induced pulmonary fibrosis. $J$. Immunol., 153, 4733-4741.

Zhu, Z., Homer, R.J., Wang, Z., Chen, Q., Geba, G.P., Wang, J., Zhang, Y. and Elias, J.A. (1999). Pulmonary expression of interleukin-13 causes inflammation, mucus hypersecretion, subepithelial fibrosis, physiologic abnormalities, and eotaxin production. J. Clin. Invest., 103, 779-788.

Zuo, F., Kaminski, N., Eugui, E., Allard, J., Yakhini, Z., Ben-Dor, A., Lollini, L., Morris, D., Kim, Y., DeLustro, B., Sheppard, D., Pardo, A., Selman, M. and Heller, R.A. (2002). Gene expression analysis reveals matrilysin as a key regulator of pulmonary fibrosis in mice and humans. Proc. Natl. Acad. Sci. USA, 99, 6292-6297. 